\title{
Arbeitsgemeinschaft für Verhaltensmodifíkation e.V. (AVM-D)
}

Am 23.3.96 trafen sich Vertreter der AVM-Deutschland und der AVM-Österreich in Bern mit Vertretern der neu gegründe-ten AVM-Schweiz.

Die Ak-Leiter- und Lehrtherapeutenkonferenz am 30.3.96 beschäftigte sich mit «Vorschlägen zur Änderung des Aus-bildungsmodells im Rahmen der Anerkennung der AVM als akkreditiertes Ausbildungsprogramm».

Die umfangreichen Vorbereitungen wurden von den Vorstandsmitgliedern in Zusammenarbeit mit der AWANK übernommen.

Der Arbeitskreis Bamberg bietet einen Workshop am 20./21.

Mi 1996 in Bamberg, Psychologische Gemeinschaftspraxis,

Pfahlplätzchen 6, mit Herrn Dipl.-Psych. Dr. W. Dorrmann an:

«Therapeutische Interventionen bei Klienten/Patienten mit

Selbsttötungsabsicht.»

Der Workshop ist als Trainingskurs ausgeschrieben und findet

jeweils von 9.00-16.00 Uhr statt.

Inhalte:

Voraussetzungen beim Therapeuten für die Arbeit mit Suizidalen

Identifikation von Selbsttötungsrisiken im Gespräch

Strategien und Techniken im Umgang mit Selbsttötungs-absichten

Probleme der stationären Einweisung und rechtliche Aspekte

5. Übliche Fehler und riskante Interventionen.

Anmeldungen sind zu richten an Frau Daniela Oberholz,

Pödeldorferstraße 84, D-96052 Bamberg. Der Workshop kostet

für studentische AVM-Mitglieder DM 180,-, für AVM-Mit-

glieder DM 215,- und für Nichtmitglieder DM 250,--

AVM- Geschäftsstelle:

Lehrstuhl für klinische Psychologie

c/o Frau Dagmar Frey

Universität Bamberg

Postfachl549

D-96045 Bamberg 
Arbeitsgemeinschaft für Verhaltensmodifíkation (AVM-Ö)

Informatìonsblatt für Ausbildungskandidaten

Dieses Blatt informiert über die einzelnen Gremien der AVM und deren Funktionen im Rahmen der Ausbildung. Es soil zur Erleichterung des Kennenlernens der Vereins- und Aus-bildungsstruktur und zur Vermeidung von Unklarheiten dienen.

1. Ausbildungsverantwortliches Gremium (AVG), zugleich

Wissenschaftlicher Beirat der AVM und des

Instituts für Verhaltenstherapie (Gemeinnützige Ges.mbH.)

Zuständigkeitsbereiche (vgl. Statuten §17):

Erarbeitung von Aus-, Fort- und Weiterbildungsrichtlinien und Durchführungsbestimmungen

Anpassung derselben an neue Entwicklungen und Internationale Standards

Präzisierung von Richtlinien, die Aus-, Fort- und Weiterbil-dung betreffend

Überwachung der Durchführung der Aus ${ }^{-}$, Fort- und Weiter-bildung im Rahmen der AVM

Sicherstellung und Verbesserung der Qualität der Ausbildung (Qualitätskommission)

Beratung der AVM und des Instituts für Verhaltenstherapie in der Entwicklung der Verhaltenstherapie

Kontaktperson:

Mag. Andrea Fahlböck, Wobischstraße la; A-9500 Villach

Tel. 04242-423 08 (priv); 04282-2220-59 (dienstl.)

2. Ausbildungs-Anerkennungsgremium (ANG):

Zuständigkeitsbereiche (Statuten, § 16):

Überprüfung der Erfüllung der individuellen Anforderungen für die Anerkennung von Aus- und Weiterbildungen in Verhaltenstherapie (Gesamtabschluß einer Aus- oder Weiterbil-dung)

Anrechnung von außerhalb der AVM erworbenen Qualifika-tionen für die Aus- und Weiterbildung im Rahmen der AVM nach Anrechnungskriterien des Psychotherapiegesetzes, Ver-ordnungen des Psychotherapiebeirates und Bestimmungen des Vereins

Überprüfung der Erfüllung der Anforderungskriterien für Lehrtherapeuten und Lehrbeauftragte zu deren Ernennung und Weiterbestellung

Zweite Instanz bei Einsprüchen gegen Entscheidungen der regionalen Arbeitskreisleitungen betreffend Ausbildungsan-erkennungen des 1. und 2.

Ausbildungsabschnittes

Kontaktperson:

Dr. Berthold Judendorfer, Landesnervenklinik Salzburg, Ignaz-

Harrer-Straße 79; A-5020 Salzburg

Tel. 0662-4483-0 (Vermittlung)

K A R. G E R (C) 1996 S. Karger GmbH, Freiburg

VI \ Fax (07 61) 4520714

3. Leitung der Regionalen Arbeitskreise:

Zuständigkeitsbereiche (Statuten, §19):

Anwerben von Lehrtherapeuten

Unterstützung der Ausbildungskandidaten in alien Belangen der Ausbildung

Kontrolle der ordnungsgemäßen Abwicklung von Prüfungen und Abschlußvorträgen

Unterstützung des Vorstandes bei der Suche nach Prakti-kumseinrichtungen

Überprüfung der Erfordernisse für den Abschluß des 1. und 2. Ausbildungsabschnittes

Abhaltung regelmäßig stattfindender Plenarsitzungen; Führung der Anwesenheitslisten

Informationsaustausch von und zu den Ausbildungskandidaten an den Vorstand und andere Gremien des Vereins.

Kontaktpersonen:

Werden jährlich vom jeweiligen Arbeitskreis gewählt

4. Vorstand der AVM:

Zuständigkeitsbereiche/'Ausbildung (Statuten, § 12):

Letztentscheidung in alien Fragen der Ausbildung und Anerkennung

Beschlußfassung von Ausbildungsrichtlinien und deren Mo-difikation

Aussprechen der Anerkennung der Aus- und Weiterbildun-gen in Verhaltenstherapie

Vertretung der AVM nach außen und innen

Förderung der Entwicklung des Vereins und der Verhaltenstherapie

Kontaktperson:

Geschäftsstelle der AVM; p.A. Mag. Tatjana Rasbortschan; Airportcenter-Tradepoint; Kasernenstraße 3b; A-5073 Wals; Tel/Fax: 0662-854138 
5. Ethikkommission der AVM:

Funktionsbereiche (Statuten, § 22):

Erarbeitung berufsethischer Standards für Verhaltensthera-peutlnnen und Lehrpersonen

Überwachung derselben und

Uberprüfung/Feststellung von Verstößen gegen diese

4. Weiterleitung der Ergebnisse an den Vorstand

Kontaktperson:

Mag. Ilona Herbst, Wimmergasse 15/13; A-1050 Wien

6. Geschäftsstelle der AVM:

Funktionsbereiche:

Zentrale Anlauf- und Auskunftstelle für alle Vereinsangele-genheiten

Drehscheibe für Informationen von und zu Mitgliedern, Gremien und anderen Institutionen

Führung der laufenden Geschäfte der AVM zusammen mit dem Vorstand

Kontaktperson:

Mag. Tatjana Rasbortschan; Airportcenter-Tradepoint; Kaser-

nenstraße 3b; A-5073 Wals; Tel/Fax: 0662-854138

7. Geschäftsführer des Instituts für Verhaltenstherapie:

Funktionsbereiche:

Organisation und Durchführung von Ausbildungsveranstal-tungen der AVM

Annahme und Weitergabe von Informationen betreffend Aus-bildung in Verhaltenstherapie Kooperation mit den Gremien des Vereins und

Führung der Geschäfte des Instituts für Verhaltenstherapie (Gemeinnützige Ges.mbH.)

Geschäftsführer:

Dr. Gernot Ottenbacher, Hettwerstraße 8, A-5020 Salzburg,

Tel. $0662-874603$ Mag. Fahlböck

Dr. Laireiter

Mitteilungen der Verbände

Information by Behavior Therapy Associations

Ein Klassiker in Neuauflage 
Herausgeber: Harald J. Freyberger, Rolf-Dieter Stieglitz 
Kompendium der Psychiatrie und Psychotherapie

Begründet von Th. Spoerri

10., vollständig neu bearbejtete und

erweiterte Auflage, orient; erí an der ICD-10 


Miteinem stark veränderten Konzept, neuen Herausgebern und Autoren erscheint das von Th. Spoerri 1961 begründete und 1984 von H. Feldmann revidierte Kompendium jetzt in seiner 10. Auflage. Die immense Weiterentwicklung der theoretischen, me-thodologischen und therapeutischen Grund-lagen in der Psychiatrie und Psychotherapie hat den Verlag und die Herausgeber dazu bewogen, die 5 einzelnen Abschnitte mit den insgesamt 37 Kapitein von verschiedenen Autoren bearbeiten zu lassen, urn so der fortschre;tenden Spezialisierung und Differenzierung des Fachgebietes gerechtzu werden. Konzeptionell orientiert sich diese Neuauflage mit der Trennung von deskr $i p{ }_{j}{ }_{i}$-phänomenologischen und ätiolog $;$ schpatho-genetischen Betrachtungsebenen an der ICD-10, deren veränderte Sichtweise sich auch ¡m europäischen Raum durchgesetzt hat. Dieses umfassende Kompendium ist Ärzten, Medizinstudenten und Psychothera-peuten ein unentbehrlicher Begleiter und bietet als Lehrbuch, Lernbuch und Nach-schlagewerk Wissen auf dem neuesten Stand. 
Inhalt

Allgemeine Gru $\pi$ dlagen

Psychiatrische Untersuchung und Befunder-hebung $\cdot$ Klassifikation und diagnostischer Prozess $\cdot$ Psychiatrische Epidemiologie Störungsgruppen

Organische (und symptomat;sche) psychi-sche Störungen · Störungen durch psy-chotrope Substanzen · Schizophrenien und wahnhafte Störungen · Affektive Störungen

- $\quad$ Neurotische, somatoforme und Bela-

stungsstörungen · Psychosomat; $;$ sche und

verwandte Störungen · Sexuelle Störungen

- Persönlichkeits- und Verhaltensstörungen

Erwachsener - Inî̉elligenzminderung · Psy-

chische Störungen des Kindes- und Jugend-

alters

Therapieverfahren

Psychiatrische Therapieverfahren: Überblick

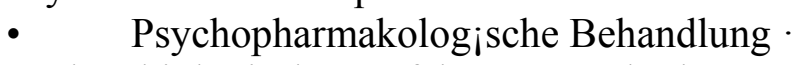

Andere biologische Verfahren · Psychotherapeutisches Gespräch und Beratung · Psychoanalytische und andere $t_{i}$ efenpsychologische Verfahren · Verhaltenstherapie · 
Gesprächspsychotherapie $\cdot$ Psychoanalyt $;$-sche Gruppenpsychotherapie und verwandte Verfahren $\cdot$ Verhaltenstherapeut $j$ sche Gruppenpsychotherapie $\cdot$ Paarund Fami-lientherapie · Andere psychotherapeutische Verfahren · Soziotherapie · Psychoeduka-tion, Patientenratgeber und Selbsth;lfe-manuale

Psychopharmakolog ${ }_{j}$ sche Not-falltherapie - Kurzpsychotherapie und Krisenintervention · Psychotherapeutische Ausbildung und Supervision Anwe $\pi$ dungsbereich

Sozialpsychiatrie und gemeindenahe Ver-sorgung $\cdot$ Forensische Psychiatrie $\cdot$ Gero $\cap$-topsychiatrie $\cdot$ Psychosomatische Mediz $j n$ einschliessl $i c h$ Grundzüge der Neurosenleh-re $\cdot$ Konsiliarpsychiatrie

Spezielle Aspekte

Therapie- und verlaufsrelevante Faktore $\cap$ psychiatrischer Störungen · Mortalität und Sujzidalität bei psychischen Störungen Eth $j k$ in der Psychiatrie

I $\pi$ teressengebiete: Psychiatrie, Psychologie; Psychoanalyse, Psychopathologie, Psychosomatik, Psychotherapie 
Kompendium der Psychiatrie und Psychotherapie Begründet von Th. Spoerri

10., vollständig neu bearbe ;tete und erweiterte Auflage

Herausgeber: Freyberger, H.J. (Lübeck/Bonn); Stieglitz, R.-D. (Freiburg)

XVI + 578 S., 14 Abb., 148 Tab., broschiert, 1996

CHF 40.-/DEM 48.-/USD 35.00

Pre;sänderungen vorbehalten

DEM-Preis nur $\mathrm{f}$ ür Deutschland, USD-Preis nur für USA

ISBN 3-8055-6173-3

KAHGEIl

Bitte $s \beta n d \beta n$ Sie:

- Expl.: Kompendium der Psychiatrie und Psychotherapie

10., vollständig neu bearbe;tete und erweiterte Auflage Herausgeber: Freyberger, H.J. (Lübeck/Bonn); Stieglitz, R.D. (Freiburg) CHF 40.-/DEM 48.-/USD 35.00 ISBN 3-8055-6173-3

$\propto$ Informat;onen zu Karger Publikat;onen in verwandten Fachgebieten 
Zahlung:

a Scheck beiliegend

a Rechnung

$\alpha$ Kred;tkarte (DEM werden in CHF belastet)

$a$ American Express $a$ Visa

o MasterCard o Eurocard

Karte-Nr.: 
a Diners

Gültig bis:

Name/Adresse:

Bei Vorauszahlung Porto und Verpackung zu Lasten des Verlags 
